\title{
NEW DEVELOPMENT OF HYPERGAM AND ITS TEST OF PERFORMANCE FOR $\gamma$-RAY SPECTRUM ANALYSIS
}

\author{
B.G. PARK ${ }^{1 *}$, H.D. CHOI ${ }^{1}$, and C.S. PARK ${ }^{2}$ \\ ${ }^{1}$ Department of Nuclear Engineering, Seoul National University \\ San 56-1 Shilim-Dong, Kwanak-Gu, Seoul, 151-744, Korea \\ ${ }^{2}$ Korea Institute of Nuclear Safety \\ 62 Gwahak-Ro, Yuseong-Gu, Daejeon 305-338, Korea \\ ${ }^{*}$ Corresponding author. E-mail : vandegra@plaza.snu.ac.kr
}

Received November 09, 2011

Accepted for Publication June 14, 2012

The HyperGam program was developed for the analysis of complex HPGe $\gamma$-ray spectra. The previous version of HyperGam was mainly limited to the analysis of $\gamma$-ray peaks and the manual logging of the result. In this study, it is specifically developed into a tool for the isotopic analysis of spectra. The newly developed features include nuclide identification and activity determination. An algorithm for nuclide identification was developed to identify the peaks in the spectrum by considering the yield, efficiency, energy and peak area for the $\gamma$-ray lines emitted from the radionuclide. The detailed performance of nuclide identification and activity determination was accessed using the IAEA 2002 set of test spectra. By analyzing the test spectra, the numbers of radionuclides identified truly (true hit), falsely (false hit) or missed (misses) were counted and compared with the results from the IAEA 2002 tests. The determined activities of the radionuclides were also compared for four test spectra of several samples. The result of the performance test is promising in comparison with those of the well-known software packages for $\gamma$-ray spectrum analysis.

KEYWORDS : $\gamma$-ray Spectrometry; Nuclide Identification; Activity Determination; HPGe; Spectral Analysis

\section{INTRODUCTION}

$\gamma$-ray spectroscopy is used in a wide range of applications such as radionuclide metrology, NAA (Neutron Activation Analysis), ERM (Environmental Radiation Monitoring) and nuclear material analysis. There are diverse and practical software packages, useful or developed commercially for the analysis of $\gamma$-ray spectra, e.g., Anges, GammaVision, Gamma-W, Genie2000, Hyperlab, and Interwinner. However, from the viewpoint of the user, some of these software packages are closely coupled with the specific hardware devices and there is a limitation in the level of user-control over the peak analysis, nuclide identification, activity determination, and various correction routines. Furthermore the tailoring or improving of such codes, in such areas as checking the results of the analysis process or modifying the style of the analysis report, is typically not open to the user. Meanwhile, from the results of the recent IAEA test [1] which estimated the performance of various widely available and commercial software packages for the analysis of low-level $\gamma$-ray spectrometry, it was reported that most packages showed reasonable results in determining the peak area and energy, but none of these programs achieved a reasonable level of perfor- mance in terms of the most crucial criteria, such as nuclide identification and determination of activity [1].

In this context, we have been developing the HyperGam program for $\gamma$-ray spectrum analysis based on the HYPERMET code [2]. The main features of HyperGam include a graphical user interface, semi-automatic algorithm for $\gamma$-ray peak analysis, system non-linearity calibration and Doppler-broadened peak analysis. These were discussed in previous papers $[3,4]$. Several functions were developed recently and incorporated into HyperGam for the purpose of applying it to environmental radiation monitoring. The new features include MCA emulation, network communication and peak monitoring [5]. MCA emulation is the function used to control the ADC interface module and transfer the spectrum data to memory in HyperGam. This function was written in $\mathrm{C}++$ language based on the user programming libraries provided by the vendors [6]. The network communication function allows for the control of remote detection devices which are connected to the server computer in the network. In the mode of peak monitoring, the analysis of the detected peaks and monitoring of the peak-area and peak-width are done in near real-time during the radiation detection procedure. The previous version of HyperGam adopted 
the graphical user interface in the MS-Windows system and its basic functions were limited to such tasks as the analysis of the peak energy and area. Therefore, it was difficult for the user to identify radionuclides and determine their activities, since this involved manually logging the analysis results and subjectively judging the data by referring to the nuclear data. In this study, an appropriate nuclear data library was formed and an algorithm was developed to identify the radionuclides in the spectrum. The algorithm of nuclide identification attempted to achieve low numbers of missing nuclides (the number of missed radionuclides known to be present in the sample) and of false hits (the number of radionuclides determined but known not to be present in the sample). The IAEA 2002 test spectra [7] were used to evaluate the performance in the identification of the nuclides and determination of their activities. The method of using efficiency-based calibration was adopted to determine the activities of the radionuclides identified in the test spectra. The results of the assessment are described.

\section{ALGORITHM OF NUCLIDE IDENTIFICATION}

The analysis of the $\gamma$-ray spectrum began by detecting the peaks and determining their energy and area. The work of identifying the radionuclide which emitted the gammaray corresponding to a peak in the spectrum is required and should be done in a consistent and reliable manner. This process is, in most cases, based on a comparison of the result of peak analysis with the data in a nuclear library. A typical algorithm of nuclide identification is based on a method of determining the closest radionuclide by matching the peak energy with the library one [8]. In this method, the process of identification is simple and fast, but usually leads to unreliable results in the case of a complicated spectrum. Another algorithm [9] which identifies radionuclides by considering their activities as well as the peak energies is effective when the peak detection efficiencies are available. The previous version of HyperGam had a limitation in its ability to identify nuclides, since it was developed for the real-time monitoring of ambient gamma radiation, in which case it is difficult to know the detection efficiency due to the unknown geometry of the sources [5]. Therefore, a new algorithm for HyperGam was developed to identify the radionuclides in the measurements where the geometry of the sources is well-defined, e.g. measurement of $\gamma$-rays from standard reference radioisotopes or fixed samples. In the new algorithm, the peaks on the spectrum are identified by considering a set of the energy, emission probability, detection efficiency, and peak area of the gamma-ray emitted from the candidate radionuclides.

Before beginning the nuclide identification process, the critical level $\left(L_{C}\right)$ and detection limit $\left(L_{D}\right)$ of a peak are calculated based on Currie's formulae [10]. When the net area of a peak is less than the detection limit, it is excluded from the next processes of nuclide identification and determination of the activity. The critical level and detection limit are defined with probabilities of less than $5 \%$ for false-negative and false-positive, respectively. Here, the background area of a peak is calculated by integrating the step-background and smooth-background derived from HyperGam's analysis result. The step background $\left(B_{S T B}\right)$ and smooth background $\left(B_{S}\right)$ in channel $J$ (or $\left.x\right)$ are described by:

$$
B_{S T B}(x)=\Sigma \frac{1}{2} \operatorname{erfc}(x / \sigma), \quad B_{S}(J)=a+b J+c J^{2}
$$

where $\Sigma$ is the step amplitude, $\sigma$ is the noise width, and $x$ is defined as $J$ (channel) $-x_{p}$ (peak channel) [2]. The background area is calculated by integrating the two background terms,

$$
\begin{aligned}
N_{B}=\int_{-1.96 \sigma_{p}}^{+1.96 \sigma_{p}} B_{S T B}(x) d x+\int_{x_{p}-1.96 \sigma_{p}}^{x_{p}+1.96 \sigma_{p}} B_{S}(J) d J \\
\quad=1.96 \sigma_{p} \Sigma+3.92 \sigma_{p}\left[a+b x_{p}+\frac{c}{3}\left\{3 x_{p}^{2}+\left(1.96 \sigma_{p}\right)^{2}\right\}\right]
\end{aligned}
$$

where $\sigma_{p}$ is the Gaussian standard deviation of the peak and the noise width is taken as $\sigma=\sqrt{2} \sigma_{p}$. If the net area of the peak is less than $L_{D}$ or $L_{C}$, the $\alpha$ - or $\beta$-error is calculated respectively and referenced in the final report of HyperGam. The $\alpha$-error and $\beta$-error are calculated by:

$$
\begin{gathered}
\alpha=\int_{V_{p}}^{\infty} \frac{1}{\sqrt{2 \pi} \sigma_{B}} \exp \left(-\frac{x^{2}}{2 \sigma_{B}^{2}}\right) d x=\frac{1}{2} \operatorname{erfc}\left(\frac{N_{p}}{\sqrt{2} \sigma_{B}}\right) \\
\beta=\int_{-\infty}^{L_{C}} \frac{1}{\sqrt{2 \pi} \sigma_{p}} \exp \left\{-\frac{\left(x-N_{p}\right)^{2}}{2 \sigma_{p}^{2}}\right\} d x=\frac{1}{2} \operatorname{erfc}\left(\frac{N_{p}-L_{C}}{\sqrt{2} \sigma_{p}}\right)
\end{gathered}
$$

where $\sigma_{B}=\sqrt{2 N_{B}}$ and $N_{p}$ is the net area of the peak.

Fig. 1 summarizes the overall procedure for nuclide identification. The nuclide identification is performed in two stages.

In the $1^{\text {st }}$ stage, HyperGam matches the radionuclides with a peak in the spectrum by using a simple library [11]. The radionuclide data of the simple library was taken from the Table of Isotopes [12] and EML (Environmental Measurement Laboratory) Report [13]. The library includes the $\gamma$-emitters which are fission products in a reactor accident as well as artificial or naturally occurring radionuclides. After loading the library, the peak analysis data of the $\gamma$-ray spectrum is read-in for the isotope identification process. In the preliminary search, primary candidate radionuclides having $\gamma$-ray energies similar to the analyzed peak energy are selected. The CM (CandidateMatching) matrix is a list of the primary candidate radionuclides in the order of the energy of their peaks, and the NM (Nuclide-Matching) matrix is the reallocated one from the CM matrix in the order of the $(\mathrm{Z}, \mathrm{A})$ of the library radionuclides. Using these matrices, the following three 


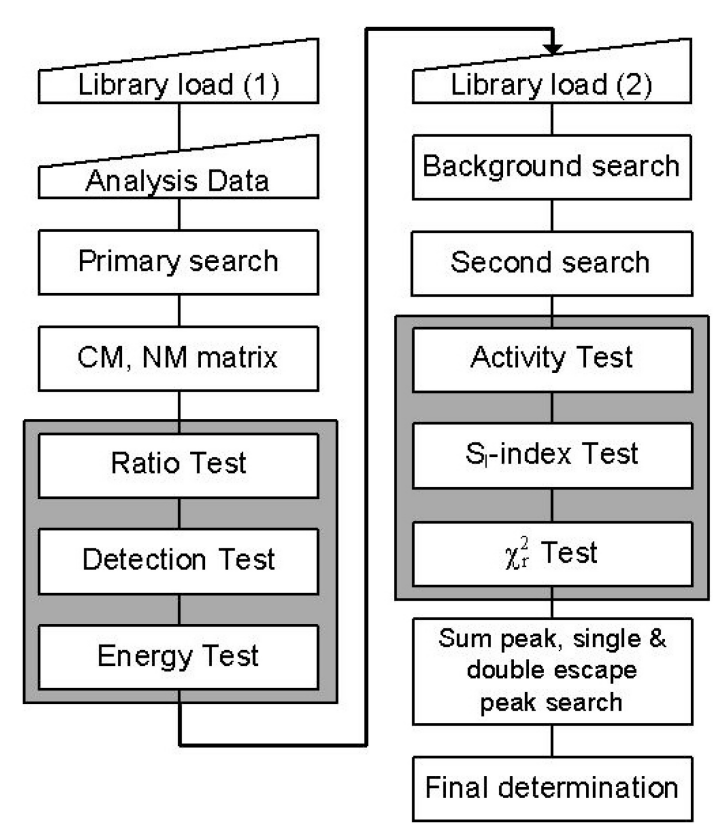

Fig. 1. Flow Chart of the Nuclide Identification Algorithm.

tests are conducted: (i) the 'Ratio test' is performed based on the crude assumption that the ratios of the peak area to the detection efficiency and emission probability are identical for the multiple gamma rays emitted from the same nuclide. (ii) The 'Detection test' considers how many peaks are detected among those lines listed in the $\gamma$-ray library, and also how high the emission probabilities of the detected peaks are. (iii) The 'Energy test' considers the energy difference between the measured peaks and the library ones. Each test generates scores of candidate radionuclides and the radionuclide with the highest score is selected. By means of the scores from the three tests, the probable candidate nuclides are listed and the first step list of the most probable nuclides is obtained.

The $2^{\text {nd }}$ stage performs a sophisticated identification and quantitative analysis by using an expanded library. The main purpose of the $2^{\text {nd }}$ stage is to minimize the number of missing nuclides and the number of false hits. Repeated searches of the candidate radionuclide based on the expanded library and tests based on the confidence of the candidate radionuclide are conducted at this stage. The expanded library includes 150 radionuclides and their gamma-ray lines. The library is generated from the Nudat 2.5 data file for each radionuclide [14]. The radionuclides in the library are classified based on their origins from the natural decay chains (the uranium, actinium and thorium series), ${ }^{241} \mathrm{Pu}$ decay chain, reactor fuel cycle products, fission products, fall-out, activation in a reactor (inside or surrounding of a reactor, or for isotope production and application), activation in an accelerator (mainly for isotope production and application) and other activations. In the next step, if the peak information of background spectrum which has been measured under the same condition as the sample spectrum is available, the identification of a peak for background peak is performed by considering both the peak energy and the count rate. The match between a peak on the background spectrum and one on the sample spectrum was judged based on the two z-scores,

$$
z_{x}=\frac{x_{i}-x_{j}}{\sqrt{\sigma_{i}^{2}+\sigma_{j}^{2}}}
$$

where $x$ is the energy or the count rate of the peak, $\sigma$ is the standard deviation of $x, i$ and $j$ are the indices for the background quantity and the sample one, respectively. If the z-scores of the energy and the count rate are below the criteria, the peak on the sample spectrum is identified as a background peak. The 'second search' step is performed in the case where a peak of uncertain identification among the matched peaks in the first stage is chosen as an unidentified peak by considering the number of matched peaks for the radionuclide and z-score of the activity based on the peak. The z-score of the activity is calculated similarly by using the eq. (5). Here $x$ is the activity, $i$ and $j$ are the indices for the quantity of the peak and of the nuclide, respectively. The candidate radionuclides are selected again by using the expanded library. The next three tests are conducted for the candidate radionuclides: (i) the 'Activity test' identifies the radionuclide of the unidentified peak by comparing the activity of the radionuclide determined in the $1^{\text {st }}$ stage with the tentative activity which is calculated based on the assumed identity of the peak emitted by the radionuclide. If the difference in activity is below the criteria, the radionuclide is assigned to the unidentified peak. (ii) The ' $\mathrm{S}_{\mathrm{I}}$-index test' is performed to quantify a measure of the completeness of the identification. The fraction of the detected lines' emission probabilities to the overall sum of those of the lines emitted from the candidate radionuclide is calculated. The index value gives more weight to the peak matching to that of higher emission probability. It is expressed as the $\mathrm{S}_{\mathrm{I}}$-index for nuclide- $i$ :

$S_{I, i}=\sum_{j=1}^{n}$ (emission probability $)_{j, i} / \sum_{k=1}^{m}$ (emission probability $)_{k, i}(6)$ where $j$ is the running index of the sum over the detected peaks and $k$ is that of the sum over the library peaks of nuclide- $i$. The $\mathrm{S}_{\mathrm{I}}$-index is between 0 (no library-peaks detected) and 1 (all library-peaks detected). From empirical experience, the candidate nuclide is regarded as being below the acceptable level for complete detection when the $\mathrm{S}_{\mathrm{I}}$-index of the radionuclide is below 0.4. In that case, the candidate nuclide is excluded from the identification. (iii) The ' $\chi_{\mathrm{r}}^{2}$-index test' is conducted to evaluate a candidate among multiple $\gamma$-ray emitters by calculating the statistical index of the library $\gamma$-rays that are undetected. The reduced $\chi^{2}$ value for nuclide- $i$ is defined by:

$$
\chi_{r, i}^{2}=\frac{1}{L-1} \sum_{u=1}^{L}\left(N_{u} / \sigma_{u}\right)^{2}
$$


where $L$ is the number of undetected peaks of nuclide- $i$, $N_{u}$ is the expected area of undetected peak $u$ if the peak is ever detected, and $\sigma_{u}$ is the combined uncertainty obtained from those of the net peak area and background area. If there are two or more candidate radionuclides corresponding to the unidentified peak, the radionuclide of the least $\chi^{2}$ value is assigned. When the $\chi^{2}$ values are larger than 1.0 for all of the candidate radionuclides, the peak is classified as an unidentified peak. After these three tests, the true coincidence sum peaks, single and double escape peaks and interfered peaks are checked for the identified nuclides. Those radionuclides that pass all of the tests are regarded as the final identified radionuclides.

The activity and MDA (Minimum Detectable Activity) [15] of the identified radionuclides are determined by considering all of the detected peaks. When the number of detected peaks of the identified nuclide is $n$ and the activity based on the $i$-th detected peak is $A_{i} \pm \Delta A_{i}$, the nuclide activity and its external and internal uncertainties are given by [16]

$$
\begin{aligned}
& \text { nuclide activity: } \quad A_{\text {nuclide }}=\frac{\sum_{i=1}^{n} w_{i} \cdot A_{i}}{\sum_{i=1}^{n} w_{i}} \\
& \text { external uncertainty: } \Delta A_{\text {nuclide }}=\sqrt{\frac{\sum_{i=1}^{n} w_{i}\left(A_{i}-A_{\text {nuclide }}\right)^{2}}{(n-1) \sum_{i=1}^{n} w_{i}}}
\end{aligned}
$$

$$
\text { internal uncertainty: } \Delta A_{\text {nuclide }}^{*}=\frac{1}{\sqrt{\sum_{i=1}^{n} w_{i}}}
$$

where $w_{i}$ is defined as $1 /\left(\Delta A_{i}\right)^{2}$. The larger value of $\Delta A_{\text {nuclide }}$ and $\Delta A^{*}{ }_{\text {nuclide }}$ is used for the reported uncertainty of the activity. The MDA of the radionuclide is taken from the least MDA value of those of the detected peaks.

The results of the peak analysis and list of the identified radionuclides are reported in the final identification report, an example of which is shown in Fig. 2. The report contains the name of the spectrum file, counting time, nuclide library used, efficiency calibration coefficients, sensitivity of the z-score, and list of identified nuclides, background peaks, identified peaks and unidentified peaks.

\section{PERFORMANCE TEST}

The performance of the nuclide identification algorithm in HyperGam was tested by using the IAEA 2002 test spectra [7]. The IAEA 2002 test spectra were prepared for low-level $\gamma$-ray spectrometry and one of the tests focused on the analysis of spectra with small area peaks due to low activity level and/or limited counting time. Other issues which were noted to be specifically relevant in the test spectra were self-attenuation in the voluminous source, coincidence summing, determination of detection efficiency at low gamma-ray energies below $100 \mathrm{keV}$, background subtraction and nuclide identification [1]. Three kinds of spectra - background spectra, calibration spectra and test spectra - were used in this study. A detailed description of the test spectra, the test procedure and the results of the tests are found in the references $[1,7]$, while a brief summary is given in this paper to provide an overview of the present performance test. A list of the test spectra is shown in Table 1. Two HPGe detectors were employed in the IAEA test spectra: one of 33\% relative efficiency, denoted "SMALL", and the other of $96.3 \%$ relative efficiency, denoted "BIG" in Table 1 . Mixed radionuclide sources were used to obtain the spectra : MARICSMALL, PICBIG $\left({ }^{241} \mathrm{Am},{ }^{109} \mathrm{Cd},{ }^{57} \mathrm{Co},{ }^{137} \mathrm{Cs}\right.$, etc. $)$, MARITSMALL60 and PITBIG60 $\left({ }^{22} \mathrm{Na},{ }^{51} \mathrm{Cr},{ }^{60} \mathrm{Co},{ }^{133} \mathrm{Ba}\right.$ and $\left.{ }^{152} \mathrm{Eu}\right)$. The MIX1EQ, MIX1NEQ and MIX2NEQ6 spectra were obtained by measuring a source of powdered uranium and thorium ores. The IAEA 2002 test spectra were used in an intercomparison of the commercially available software packages, i.e. Anges 1.0, GammaVision 5.3, Gamma-W 1.68 for Windows, Genie2000 2.1, Hyperlab 2002.3.2.18, InterWinner 5.0 and UniSampo 1.97. The same method and procedures as those used in the intercomparison in reference [1] were used for HyperGam in this study. Admittedly the present test could not be performed under the same conditions as those in ref. [1], for example, the testing time, place and personnel. The peak search parameters of HyperGam were set to the default values for all of the test spectra in this study. When using HyperGam, an approximate estimate of peak widths at the two channel positions is required for the user input parameters. These are used only as the initial guess values in the search and fitting process of the peak analysis which is proceeded afterwards by the 'semi-automatic' way for all the peaks on the spectrum.

\subsection{Library Comparison and Analysis of Calibration Spectra}

In the starting test, the nuclide library of HyperGam and commercial programs were compared. The "Laboratoire National Henri Becquerel" data from "Nucleide 2000" was used as the reference data [1]. The values of the energy and yield of each line of the expanded library of HyperGam were consistent with the reference values. Due to space limitations, the full results of the library comparison are omitted in this paper. The determination of the detection efficiency and the calibration of the energy non-linearity for the "SMALL" and "BIG" detectors were done by using the calibration spectra MARICSMALL and PICBIG, respectively. In the energy range of $59.5-1836.1 \mathrm{keV}$, 16 peaks of the MARICSMALL spectrum and 20 peaks of the PICBIG spectrum were analyzed in the calibration fitting. The channel-energy calibration was fitted with a 


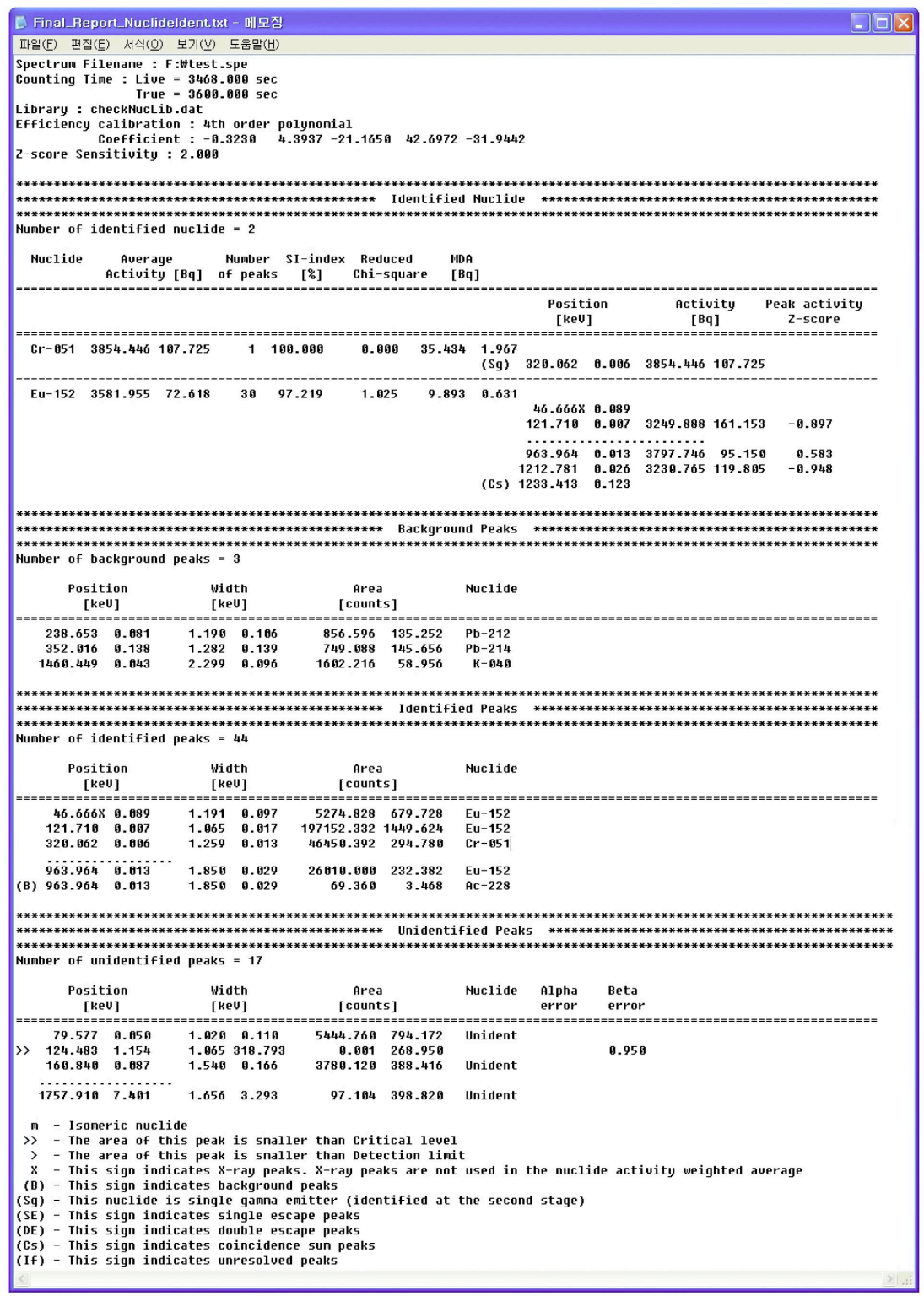

Fig. 2. Final Report of HyperGam.

line and the full energy peak efficiency was fitted with a 4 th order polynomial. The non-linearity data of the channel-energy calibration were reproduced with a 4 th order polynomial by comparing the measured peak position with the reference energy of each line which was taken from Nudat 2.5 [14]. The non-linearity and its $1 \sigma$ uncertainty are shown in Fig. 3. The maximum energy non-linearities of the "SMALL" and "BIG" detectors were 0.21 and $0.24 \mathrm{keV}$, respectively. The absolute peak efficiency curves of the two detectors are shown in Fig. 4. In this version of HyperGam, the detection efficiency curve is determined by using the manual input data of the $\gamma$-ray energy and efficiency. In this test, we considered all of the radionuclides $\left({ }^{54} \mathrm{Mn},{ }^{57} \mathrm{Co},{ }^{65} \mathrm{Zn},{ }^{88} \mathrm{Y},{ }^{109} \mathrm{Cd},{ }^{113} \mathrm{Sn}\right.$, $\left.{ }^{134} \mathrm{Cs},{ }^{137} \mathrm{Cs},{ }^{139} \mathrm{Ce},{ }^{203} \mathrm{Hg},{ }^{241} \mathrm{Am}\right)$ of the MARICSMALL and PICBIG spectra to determine the efficiency curve. For the single $\gamma$-ray emitters, the corresponding $\gamma$-ray peak was used, while for the multiple $\gamma$-ray emitters, those $\gamma$ ray peaks with an emission probability of more than $10 \%$ were used. The coincidence summing correction was not performed for the determination of the efficiency curve. As shown in Fig. 4, the efficiency curve of MARICSMALL can be, however, described well by a single curve. On the other hand, the efficiency data of the PICBIG spectrum cannot be described as a single curve. These curves show that correction routines for self-attenuation and coincidence summing are required for HyperGam in the future. The uncertainty of the fitted efficiency was calculated according 
to the reference [17] and used to determine the radionuclide activity and activity uncertainty.

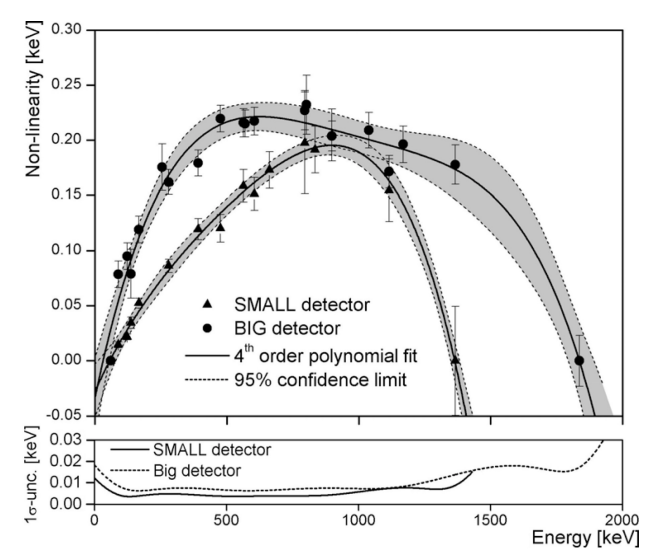

Fig. 3. Non-linearity Curves for "SMALL" Detector and "BIG" Detector.

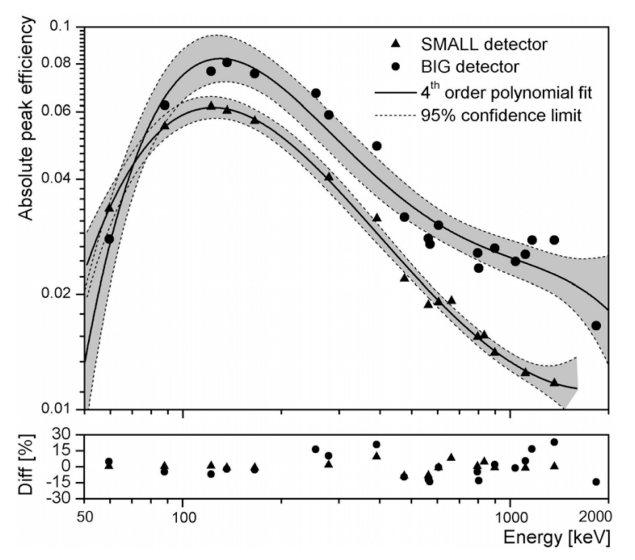

Fig. 4. Absolute Peak Efficiency Curves for "SMALL" Detector and "BIG" Detector.

\subsection{Analysis of Small Peaks and Peak Areas}

The performance of HyperGam was tested for determining the peak areas in the cases of spectra with low statistics. The MIX2NEQ6 spectrum was analyzed and the analysis results of HyperGam were compared with those of the commercial programs. The number of analyzed small peaks is related to the threshold setting of the peak search algorithm of the program. In Fig. 5, the numbers of small peaks and false hits are shown. Thirty-four small peaks and one false hit were reported by HyperGam, with the default settings. The results show that HyperGam performed similarly to other commercial programs in terms of its ability to detect small peaks on a low background. When the threshold values of "SIG" for the peak search and "RSIG" for the residual test [3] were lowered to $(3,2)$, $(2.5,2),(1,1)$, the number of small hits increased to 42 , 51 and 102, respectively, while the number of false hits increased accordingly to 3,7 , and 12. Fig. 6 shows the

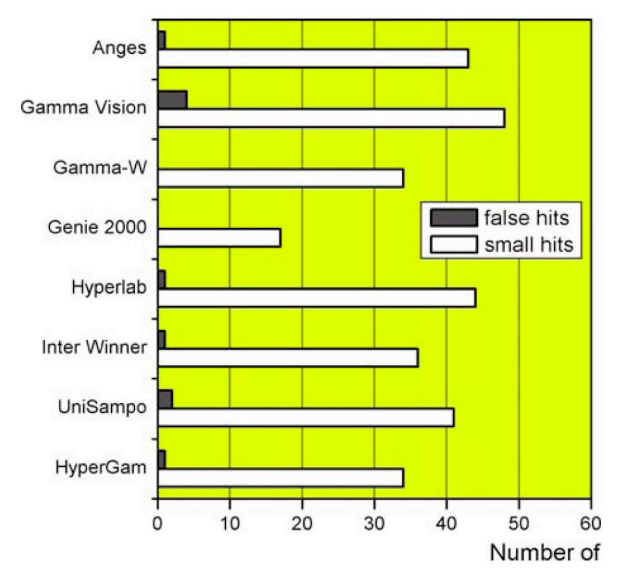

Fig. 5. Numbers of Small Peaks Detected and False Hits Reported for the MIX2NEQ6 Spectrum.

Table 1. List of the IAEA 2002 Test Spectra [7] used in this Study.

\begin{tabular}{|c|c|c|c|c|c|}
\hline Spectrum name & Detector & Geometry & Source & $\begin{array}{c}\text { Counting time } \\
\text { [min }]\end{array}$ & Note \\
\hline BGSMALLMARI & SMALL & Marinelli beaker & Water & 1871 & Background \\
\hline BGBIGPOINT & BIG & No sample & None & 600 & Background \\
\hline MARICSMALL & SMALL & Marinelli beaker, $1.15 \mathrm{~g} / \mathrm{ml}$ & Mixed source & 4091 & Calibration \\
\hline PICBIG & $\mathrm{BIG}$ & Pillbox, $1.15 \mathrm{~g} / \mathrm{ml}$ & Mixed source & 2880 & Calibration \\
\hline MARITSMALL60 & SMALL & Marinelli beaker, $1.15 \mathrm{~g} / \mathrm{ml}$ & Mixed source & 60 & Test \\
\hline PITBIG60 & $\mathrm{BIG}$ & Pillbox, $1.6 \mathrm{~g} / \mathrm{ml}$ & Mixed source & 60 & Test \\
\hline MIX1EQ & SMALL & Marinelli beaker, $1.0 \mathrm{~g} / \mathrm{ml}$ & Natural radioactivity source & 60 & Test \\
\hline MIX1NEQ & SMALL & Marinelli beaker, $1.0 \mathrm{~g} / \mathrm{ml}$ & Natural radioactivity source & 2861 & Test \\
\hline MIX2NEQ6 & SMALL & Marinelli beaker, $1.0 \mathrm{~g} / \mathrm{ml}$ & Natural radioactivity source & 6 & Test \\
\hline
\end{tabular}

\footnotetext{
${ }^{*}$ True time for measurement
} 
reduced $\chi^{2}$ values computed from the reference uncertainties only ("reference") and from the combination of both the reference and calculated uncertainties of the program ("reported") for the small peaks on a low background and all of the peaks. The reduced $\chi^{2}$ values were calculated by using the same procedures as in the IAEA 1995 test [18]. The reference uncertainties denote those of peak areas which were contained in the reference list used for the IAEA 2002 test [1], and the reported uncertainties denote those of peak areas which were determined by using HyperGam. The reference $\chi^{2}$ value reflects the accuracy of the peak area determination, and the reported $\chi^{2}$ value indicates the level of statistical control of the program. HyperGam reported reasonably good peak areas and performed better than the other programs in terms of its statistical control.

\subsection{Radionuclide Identification}

To estimate the performance in terms of the nuclide identification, the MARITSMALL60, PITBIG60, MIX1EQ, and MIX1NEQ spectra were analyzed quantitatively by HyperGam. When radionuclides known to be detectably present in the sample were identified, they were counted as 'hits'. Similarly, radionuclides known to be detectably present in the sample but not identified by the program were counted as 'misses'. Any radionuclide identified but known not to be detectably present in the sample was counted as a 'false hit'. Figs. 7 and 8 show how many peaks were reported correctly and how many false hits and misses were reported. The performances of the commercial programs [1] are also displayed for the purpose of comparison. In Fig. 7, it is shown that the MARITSMALL60 and PITBIG60 spectra are relatively simple to analyze. HyperGam, Anges, Gamma-W, and Genie 2000 identified all of the radionuclides present in the sample. On the other hand, the MIX1EQ and MIX1NEQ spectra, as shown in Fig. 8, have many $\gamma$-ray peaks, viz. the 101 and $224 \gamma$-ray peaks, respectively, according to the analysis by HyperGam and none of the programs were able to identify all of the radionuclides in the sample. HyperGam identified 13 and 16 of these 22 unknown radionuclides present in the MIX1EQ and MIX1NEQ samples, respectively.

\subsection{Activity Determination}

The activities of the identified radionuclides in the MARITSMALL60, PITBIG60, MIX1EQ, and MIX1NEQ spectra were determined by using HyperGam. The ratios of reported and certified activities for the test spectra are
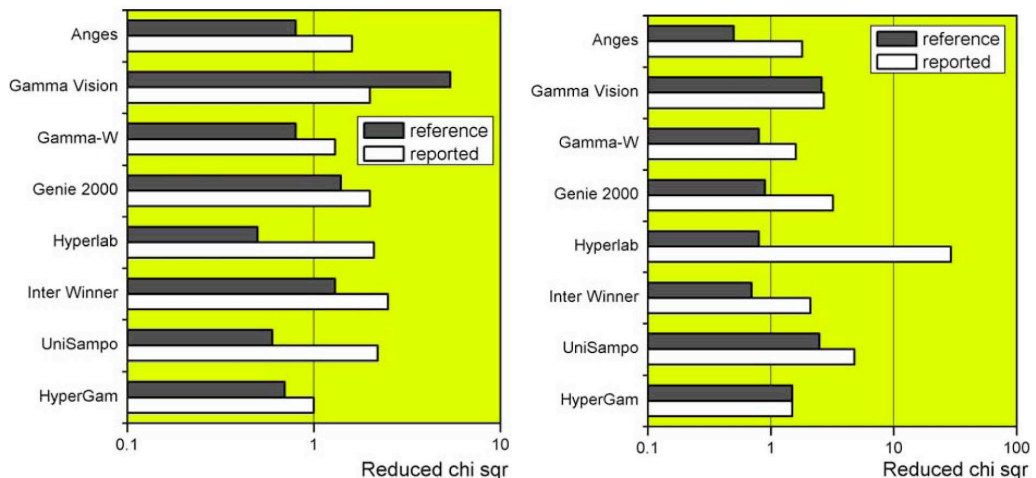

Fig. 6. Quality of Peak Area Determination and Statistical Control of the Various Programs for Small Peaks on a Low Background (Left) and for All of the Peaks (Right) in the MIX2NEQ6 Spectrum.
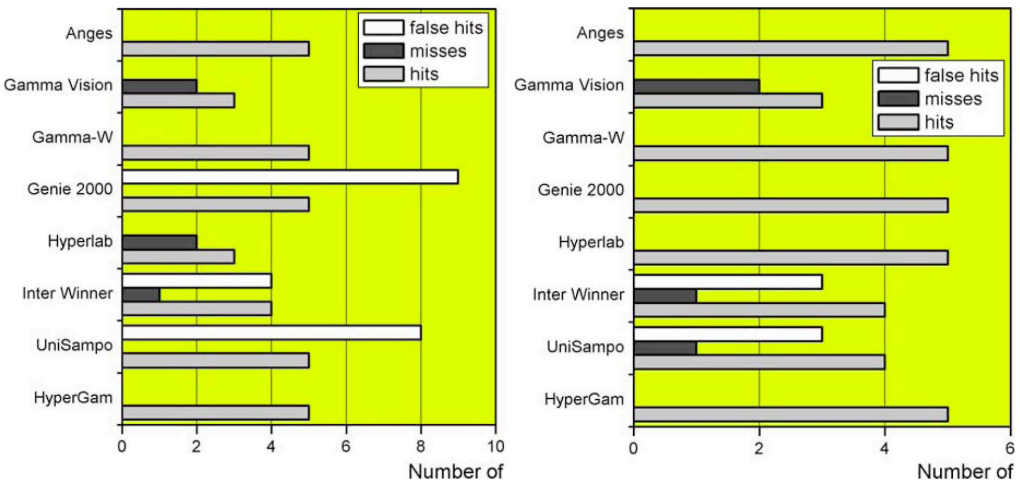

Fig. 7. Numbers of Identified Radionuclides, Misses and False Hits for the MARITSMALL60 Spectrum (Left) and for the PITBIG60 Spectrum (Right). 

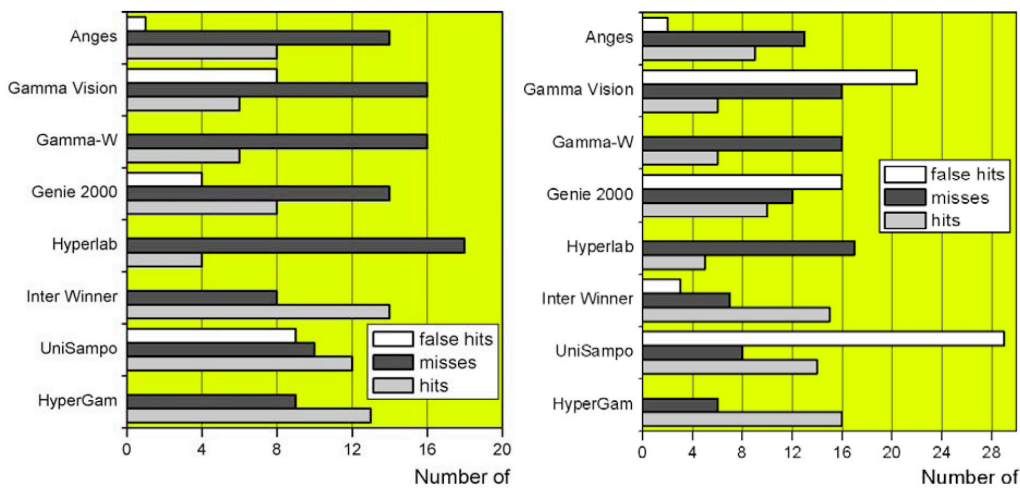

Fig. 8. Numbers of Identified Radionuclides, Misses and False hits for the MIX1EQ Spectrum (Left) and for the MIX1NEQ Spectrum (Right).
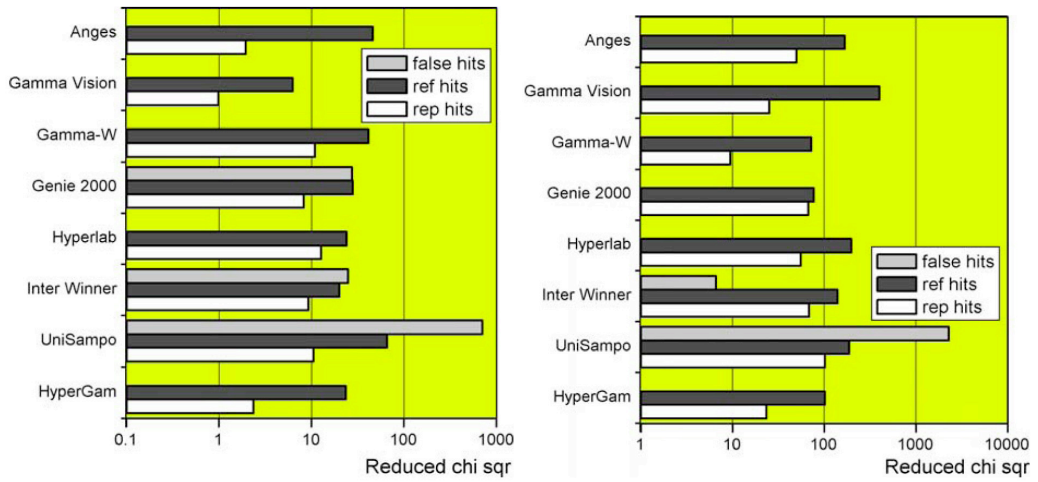

Fig. 9. The Reduced $\chi^{2}$ Values Based on the Reported Uncertainties ("False Hits" and "Rep Hits") and on the Reference Uncertainties ("Ref Hits") for the MARITSMALL60 Spectrum (Left) and PITBIG60 Spectrum (Right).

shown in Table 2. The ratios of activities lie in the range of $0.6-1.3$. As can be seen in Table 2, HyperGam failed to report ${ }^{40} \mathrm{~K}$ in the MIX1EQ and MIX1NEQ spectra. ${ }^{40} \mathrm{~K}$ was hardly present in the MIX1EQ and MIX1NEQ samples and the $1460.8 \mathrm{keV}$ peak of ${ }^{40} \mathrm{~K}$ was interfered with the $1459.2 \mathrm{keV}$ peak of ${ }^{228} \mathrm{Ac}$. None of the commercial programs properly dealt with the $1459.2-1460.8 \mathrm{keV}$ doublet. In some cases, the activity of ${ }^{40} \mathrm{~K}$ that they reported was too high (ratios were $31.9-133.3$ ), which is due to their missing the background peaks of ${ }^{228} \mathrm{Ac}$ and ${ }^{40} \mathrm{~K}$ [1]. HyperGam also failed to resolve the 1459.2 - $1460.8 \mathrm{keV}$ doublet in the MIX1EQ spectrum. The area of the doublet was corrected in HyperGam by using the background peak of ${ }^{40} \mathrm{~K}$ logged in the background search step. This peak was, however, mistaken for the $1459.2 \mathrm{keV}$ peak of ${ }^{228} \mathrm{Ac}$ in the sample. In the case of the MIX1NEQ spectrum, the doublet peak was properly decomposed by HyperGam in the peak analysis step. The $1460.8 \mathrm{keV}$ peak was matched to the background peak of ${ }^{40} \mathrm{~K}$, but its corrected area was not significant enough to identify ${ }^{40} \mathrm{~K}$ in the sample.

To compare the performance of HyperGam in the activity determination, the reduced $\chi^{2}$ value and two kinds of z-scores were calculated as well. These values are given by [1]:

$$
\begin{gathered}
\chi_{r}^{2}=\frac{1}{N} \sum_{i=1}^{N} z_{i}^{2}, \quad z_{i}=z_{r e f} \text { or } z_{\text {rep }} \\
z_{\text {ref }}=\frac{A_{\text {rep }}-A_{\text {cert }}}{s_{\text {cert }}} \text { and } z_{\text {rep }}=\frac{A_{\text {rep }}-A_{\text {cert }}}{\sqrt{s_{\text {rep }}^{2}+s_{\text {cert }}^{2}}} .
\end{gathered}
$$

where $A_{\text {rep }}$ and $A_{\text {cert }}$ are the reported and certified activities, and $s_{\text {rep }}$ and $s_{\text {cert }}$ are the reported and certified (reference) uncertainties of one standard deviation $(1 \sigma)$, respectively. The $z_{\text {rep }}$ value was also calculated for "false hits" by setting $A_{\text {cert }}$ and $s_{\text {cert }}$ to zero. The reduced $\chi^{2}$ values for the MARITSMALL60 and PITBIG60 spectra are shown in Fig. 9. The reduced $\chi^{2}$ value based on the reference uncertainties reflects the accuracy of the activity determination. As shown in Fig. 9, HyperGam reported reasonably accurate activities, that is, the reference $\chi^{2}$ values of HyperGam are the third lowest among the testing programs. With respect to the level of statistical control which is measured by the reported $\chi^{2}$ value, HyperGam performed the third best for the MARITSMALL60 spectrum and the second best for the PITBIG60 spectrum.

The test results so far indicate that there are some remaining issues which need to be improved in the case of HyperGam. The first topic is the true coincidence sum correction in the calibration and analysis steps. The activities 
Table 2. Results of HyperGam for the Test Spectra in Terms of the Ratios of Reported and Certified Activities.

\begin{tabular}{|c|c|c|c|c|}
\hline Sample & \multicolumn{2}{|c|}{ MARITSMALL60 } & \multicolumn{2}{|c|}{ PITBIG60 } \\
\hline Radioisotope & Ratio & Error & Ratio & Error \\
\hline $\mathrm{Na}-22$ & 0.84 & 0.05 & 0.70 & 0.03 \\
\hline $\mathrm{Cr}-51$ & 1.06 & 0.06 & 1.01 & 0.05 \\
\hline $\mathrm{Co}-60$ & 0.95 & 0.04 & 0.81 & 0.03 \\
\hline Ba-133 & 0.96 & 0.05 & 0.89 & 0.10 \\
\hline Eu-152 & 0.99 & 0.03 & 0.91 & 0.02 \\
\hline Sample & \multicolumn{2}{|c|}{ MIX1EQ } & \multicolumn{2}{|c|}{ MIX1NEQ } \\
\hline Radioisotope & Ratio & Error & Ratio & Error \\
\hline $\mathrm{K}-40$ & N.D & N.D & N.D & N.D \\
\hline $\mathrm{U}-238$ & N.D & N.D & N.D & N.D \\
\hline Th-234 & 1.31 & 0.24 & 1.33 & 0.23 \\
\hline $\mathrm{Pa}-234 \mathrm{~m}$ & 1.15 & 0.12 & 1.07 & 0.15 \\
\hline Ra-226 & 0.79 & 0.15 & 0.79 & 0.36 \\
\hline $\mathrm{Pb}-214$ & 1.04 & 0.11 & 1.05 & 0.06 \\
\hline Bi-214 & 0.95 & 0.02 & 0.90 & 0.02 \\
\hline $\mathrm{Pb}-210$ & 0.62 & 0.09 & 0.76 & 0.09 \\
\hline U-235 & 1.33 & 0.18 & 1.28 & 0.03 \\
\hline Th-231 & N.D & N.D & N.D & N.D \\
\hline Ac- 227 & N.D & N.D & N.D & N.D \\
\hline Th-227 & N.D & N.D & 0.70 & 0.04 \\
\hline $\mathrm{Rn}-219$ & 0.94 & 0.24 & 1.07 & 0.09 \\
\hline $\mathrm{Pb}-211$ & N.D & N.D & 1.05 & 0.59 \\
\hline Th-232 & N.D & N.D & N.D & N.D \\
\hline Ac-228 & 0.88 & 0.03 & 0.82 & 0.04 \\
\hline Th-228 & N.D & N.D & N.D & N.D \\
\hline Ra-224 & 0.95 & 0.07 & 0.99 & 0.06 \\
\hline $\mathrm{Rn}-220$ & N.D & N.D & 1.25 & 0.27 \\
\hline $\mathrm{Pb}-212$ & 0.97 & 0.04 & 0.84 & 0.12 \\
\hline Bi-212 & 1.05 & 0.06 & 1.03 & 0.05 \\
\hline Tl-208 & 0.97 & 0.04 & 0.90 & 0.05 \\
\hline
\end{tabular}

N.D: not detected

of such radionuclides as ${ }^{22} \mathrm{Na},{ }^{60} \mathrm{Co},{ }^{133} \mathrm{Ba}$, and ${ }^{214} \mathrm{Bi}$ which have coincidence summing peaks were underestimated by HyperGam, as shown in Table 2. In particular, the activities of ${ }^{22} \mathrm{Na}$ were found to be $16 \%$ and $30 \%$ less than the certified activities for the MARITSMALL60 and PITBIG60 spectra, respectively. This was attributed to the coincident count losses of the $1274 \mathrm{keV}$ peak which is used for identification. The second one is the self-attenuation correction for voluminous samples. HyperGam reported very low radionuclide activities for the PITBIG60 spectrum, except for that of ${ }^{51} \mathrm{Cr}$. This was due to the difference in density between the calibration (PICBIG, $1.15 \mathrm{~g} / \mathrm{ml}$ ) and test (PITBIG60, $1.6 \mathrm{~g} / \mathrm{ml}$ ) samples. The third task is a routine used for automatic efficiency calibration in order to deal properly with the efficiency curve in the low energy region. The present version of HyperGam requires the user to manually calculate the detection efficiency by using the data of the analyzed $\gamma$-ray peaks and those of the nuclear library. This manual procedure could be improved in the future. There is also a problem of the activity 
determination of the single gamma emitter ${ }^{210} \mathrm{~Pb}[1]$. This is due to the extrapolation of the efficiency curve from $59 \mathrm{keV}$ down to $46 \mathrm{keV}$ as well as the effect of sample self-attenuation [1].

\section{CONCLUSIONS}

The recent developments in the $\gamma$-ray spectrum analysis program HyperGam were summarized. For isotopic and quantified analysis, an algorithm for nuclide identification and activity determination was developed and incorporated into HyperGam. The algorithm for nuclide identification is composed of two stages based on simple and expanded libraries. Both stages include the tests for nuclide identification which consider the emission probability, detection efficiency and peak area, as well as the peak energy. In addition, routines for calculating the detection limits, subtracting the background peaks and search routines for true coincidence sum peak, single and double escape peaks were added. The nuclide identification function of HyperGam was tested by using the IAEA 2002 test spectra to assess the program's performance for application in lowlevel $\gamma$-ray spectrometry. The test results were compared with those of the commercially available programs. The new version of HyperGam exhibited comparable performance in the analysis of small peaks. The sensitivity for detecting small peaks can be increased by adjusting the parameters in the peak search of HyperGam, but at the expense of reporting more false hits by including noisy peaks whose net areas are less than the detection limit. The present result indicates so far that the default values of the peak search routine in HyperGam are close to the optimum. The performance for nuclide identification was assessed by determining the numbers of hits, false hits and missing nuclides. HyperGam reported the best performance in terms of the number of hits for all of the test spectra except for the MIX1EQ spectrum. In this sense, we can conclude that HyperGam has the satisfactory ability to verify the presence of a radionuclide compared with the commercial programs. The activity of the radionuclides is determined by considering multiple information and HyperGam showed reasonable results for most radionuclides. Meanwhile, underestimation of activities which were revealed from the test results remains as an issue. This issue shows that the correction routines for coincidence summing, self-attenuation and the routine for automatic efficiency calibration are required for HyperGam in the future.

\section{ACKNOWLEDGEMENTS}

This work has been supported by Ministry of Education, Science \& Technology (MEST) of Korea, and also supported by the Brain Korea 21 project. In addition the authors would like to thank Prof. M. Blaauw for supplying us with the full results of the intercomparison.

\section{REFERENCES}

[ 1 ] D. Arnold, M. Blaauw, S. Fazinic and V.P. Kolotov, "The 2002 IAEA Intercomparison of Software for Low-level $\gamma$ ray Spectrometry," Nucl. Instr. and Meth. in Phys. Res. A, 536, pp.196-210 (2005).

[2 ] G.W. Phillips and K.W. Marlow, "Automatic Analysis of Gamma-ray Spectra from Germanium Detectors," Nucl. Instr. and Meth., 137, pp.525-536 (1976).

[ 3 ] C.S. Park, H.D. Choi, G.M. Sun and J.H. Whang, "Status of Developing HPGe $\gamma$-ray Spectrum Analysis Code HYPERGAM,” Prog. in Nucl. Energ., 50, pp.389-393 (2008).

[ 4 ] H.D. Choi, N.S. Jung and B.G. Park, "Analysis of Dopplerbroadened Peak in Thermal Neutron Induced ${ }^{10} \mathrm{~B}(\mathrm{n}, \alpha \gamma)^{7} \mathrm{Li}$ Reaction using HYPERGAM," Nucl. Eng. Technol., vol. 41, no. 1, pp.113-124 (2009).

[ 5 ] B.G. Park, C.S. Park, N.S. Jung and H.D. Choi, "Development of HYPERGAM for Ambient Gamma Radiation Monitoring," Transactions of the Korean Nuclear Society Autumn Meeting, Gyeongju, Korea, Oct. 29-30, 2009.

[6] EG\&G ORTEC, "Connections Programmer's Toolkit A11-B32,” ORTEC, Oak Ridge, TN, USA, 2002.

[ 7 ] J.M. Los Arcos, M. Blaauw, S. Fazinic and V.P. Kolotov, "The 2002 IAEA Test Spectra for Low-level $\gamma$-ray Spectrometry Software," Nucl. Instr. and Meth. in Phys. Res. A, 536, pp. 189-195 (2005).

[ 8 ] V.S. Kondrashow, Z.D. Moroz, A.A. Kolyshkin and R. Vaillancourt, "A Computer Program for the Identification of Nuclides by using Median Estimates of Peak Areas in Gammaray Spectra," Nucl. Instr. and Meth. A, 328, pp.542-546 (1993).

[9 ] R. Gunnink, H.B. Levy and J.B. Niday, "Identification and Determination of Gamma Emitters by Computer analysis of Ge(Li) Spectra," Report UCID-15140, Lawrence Radiation Laboratory, 1967.

[10] L.A. Currie, "Limits for Qualitative Detection and Quantitative Determination," Anal. Chem., 40, pp.586-593 (1968).

[11] C.S. Park, Ph.D. Thesis, “An Automatic Nuclide Identification for Ambient Gamma Radiation Monitoring by Radionuclide", Seoul National University, Seoul, Korea, 2009.

[12] R.B. Firestone, V.S. Shireley, C.M. Baglin, S.Y. Frank Chu and J. Zipkin, Table of Isotopes, $8^{\text {th }}$ ed., John Wiley \& Sons Inc., New York (1996).

[13] Environmental Measurements Laboratory, "EML Procedures Manual," Report HASL-300, U.S. Department of Energy, 1997.

[14] National Nuclear Data Center, Brookhaven National Laboratory, "Nudat2.5". Available on http:// www.nndc. bnl.gov/nudat2/

[15] G.F. Knoll, Radiation Detection and Measurement, third ed., Wiley, New York, pp.94-96 (1999).

[16] K. Debertin, "International Intercomparison of Gammaray Emission-rate Measurements by means of Germanium Spectrometers and ${ }^{152} \mathrm{Eu}$ Sources," Nucl. Instr. and Meth., 158, pp.479-486 (1979).

[17] G.M. Sun, C.S. Park and H.D. Choi, "Determination of the Prompt $\mathrm{k}_{0, \mathrm{H}}$ Factors and Partial $\gamma$-ray Production Cross Sections for B, N, Si, P, S and Cl," J. Radioanal. Nucl. Chem., 264, pp.603-616 (2005).

[18] M. Blaauw, V.O. Fernandez, P. van Espen, G. Bernasconi, R.C. Noy, H.M. Dung and N.I. Molla, "The 1995 IAEA Intercomparison of $\gamma$-ray Spectrum Analysis Software," Nucl. Instr. and Meth. in Phys. Res. A, 387, pp.416-432 (1997). 